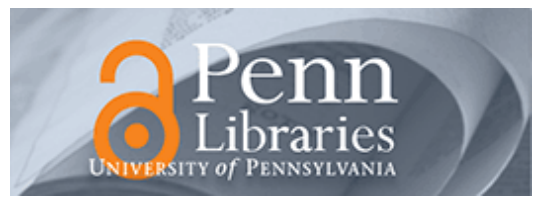

University of Pennsylvania

ScholarlyCommons

Finance Papers

Wharton Faculty Research

2008

\title{
Measuring Business Cycles: A Wavelet Analysis of Economic Time Series
}

Motohiro Yogo

University of Pennsylvania

Follow this and additional works at: https://repository.upenn.edu/fnce_papers

Part of the Econometrics Commons, and the Finance and Financial Management Commons

\section{Recommended Citation}

Yogo, M. (2008). Measuring Business Cycles: A Wavelet Analysis of Economic Time Series. Economics Letters, 100 (2), 208-212. http://dx.doi.org/10.1016/j.econlet.2008.01.008

This paper is posted at ScholarlyCommons. https://repository.upenn.edu/fnce_papers/399

For more information, please contact repository@pobox.upenn.edu. 


\title{
Measuring Business Cycles: A Wavelet Analysis of Economic Time Series
}

\author{
Abstract \\ Multiresolution wavelet analysis is a natural way to decompose an economic time series into trend, cycle, \\ and noise. The method is illustrated with GDP data. The business-cycle component of the wavelet-filtered \\ series closely resembles the series filtered by the approximate bandpass filter. \\ Disciplines \\ Econometrics | Finance and Financial Management
}




\title{
Measuring business cycles: A wavelet analysis of economic time series
}

\author{
Motohiro Yogo*
}

January 8, 2008

\begin{abstract}
Multiresolution wavelet analysis is a natural way to decompose an economic time series into trend, cycle, and noise. The method is illustrated with GDP data. The business-cycle component of the wavelet-filtered series closely resembles the series filtered by the approximate bandpass filter.
\end{abstract}

Keywords: Band-pass filter; Business cycle; Linear filter; Trend; Wavelets JEL classification: C5; E3

\section{Introduction}

Multiresolution wavelet analysis is a useful tool for studying the time and frequency properties of an economic time series. Using a wavelet filter, a time series $x(t)$ can be decomposed as

$$
x(t)=x_{0}(t)+\sum_{j=0}^{3} y_{j}(t)
$$

*Finance Department, The Wharton School, University of Pennsylvania, 3620 Locust Walk, Philadelphia, PA 19104, USA and National Bureau of Economic Research, Cambridge, MA 02138, USA. Tel.: 215-8983609; fax: 215-898-6200. E-mail address: yogo@wharton.upenn.edu. 
where $x_{0}(t)$ denotes cycles with periodicity greater than 32 periods and $y_{j}(t)$ denotes cycles with periodicity between $2^{4-j}$ and $2^{5-j}$ periods. If the sampling frequency is quarterly, $x_{0}(t)$ is the long-run trend (periodicity greater than 32 quarters). $y_{0}(t), y_{1}(t)$, and $y_{2}(t)$ are the business-cycle components (periodicity of 16-32, 8-16, and 4-8 quarters, respectively). $y_{3}(t)$ is high frequency noise (periodicity less than 4 quarters).

Applying wavelet analysis to the US real GDP in the postwar period (1947:1-2003:2),

the business-cycle component (i.e. $\left.\sum_{j=0}^{2} y_{j}(t)\right)$ closely resembles the series filtered by the approximate bandpass filter (Baxter and King, 1999). In that sense, wavelet filtering is an alternative to bandpass filtering. However, wavelet analysis provides better resolution in the time domain since wavelet basis functions are time-localized (in addition to being scalelocalized), which is useful for capturing the changing volatility of the business cycle. The wavelet-filtered series reveal that most of the business-cycle volatility before the 1960's was due to cycles of 8-16 quarters. Most of the volatility in the 1970's was due to lower frequency cycles of 16-32 quarters. Since the early 1980's, the volatility has been relatively small at all frequencies, which is consistent with recent evidence (Kim and Nelson, 1999; McConnell and Perez-Quiros, 2000).

Section 2 provides a brief overview of multiresolution wavelet analysis, highlighting properties that are relevant for the empirical application. The reader is referred to Strang and Nguyen (1997) for details. Section 3 applies the methods to study the time and frequency properties of real GDP. The MATLAB code used in the analysis is available from the author's webpage.

\section{Multiresolution wavelet analysis}

\subsection{Overview}

The design of wavelets begins with a two-channel perfect reconstruction filter bank. The lowpass and highpass analysis filters are $\left\{h_{0}(k)\right\}_{k=0}^{\widetilde{N}}$ and $\left\{h_{1}(k)\right\}_{k=0}^{N}$. The lowpass and high- 
pass synthesis filters are $\left\{f_{0}(k)\right\}_{k=0}^{N}$ and $\left\{f_{1}(k)\right\}_{k=0}^{\widetilde{N}} \cdot{ }^{1}$ The analysis scaling function $\widetilde{\phi}(t)$ is defined by the analysis dilation equation,

$$
\widetilde{\phi}(t)=\sum_{k=0}^{\widetilde{N}} 2 h_{0}(k) \widetilde{\phi}(2 t-k) .
$$

The analysis wavelet $\widetilde{w}(t)$ is defined by the analysis wavelet equation,

$$
\widetilde{w}(t)=\sum_{k=0}^{N} 2 h_{1}(k) \widetilde{\phi}(2 t-k) .
$$

The corresponding synthesis dilation and wavelet equations are

$$
\begin{aligned}
\phi(t) & =\sum_{k=0}^{N} 2 f_{0}(k) \phi(2 t-k), \\
w(t) & =\sum_{k=0}^{\tilde{N}} 2 f_{1}(k) \phi(2 t-k) .
\end{aligned}
$$

Let $\widetilde{\phi}_{j k}(t)=2^{j / 2} \widetilde{\phi}\left(2^{j} t-k\right)$ and $\widetilde{w}_{j k}(t)=2^{j / 2} \widetilde{w}\left(2^{j} t-k\right)$ be the analysis scaling function and wavelet at scale $j$ and location $k$. The corresponding synthesis scaling function and wavelet are denoted by $\phi_{j k}(t)$ and $w_{j k}(t)$. The $J$-level wavelet decomposition of a continuous time signal $x(t)$ is

$$
x(t)=\sum_{k} \widetilde{a}_{0 k} \phi_{0 k}(t)+\sum_{j=0}^{J-1} \sum_{k} \widetilde{b}_{j k} w_{j k}(t),
$$

where

$$
\begin{aligned}
\widetilde{a}_{0 k} & =\int x(t) \widetilde{\phi}_{0 k}(t) d t, \\
\widetilde{b}_{j k} & =\int x(t) \widetilde{w}_{j k}(t) d t .
\end{aligned}
$$

Let $H_{0}(\omega)$ and $H_{1}(\omega)$ be the frequency responses of the lowpass and highpass analysis

\footnotetext{
${ }^{1}$ See Strang and Nguyen (1997, Chapter 4.1) for the conditions that the filters must satisfy for perfect reconstruction.
} 
filters. The filter relating the scaling coefficients $\widetilde{a}_{0 k}$ to the original signal has a frequency response

$$
A_{0}(\omega)=\prod_{l=0}^{J-1} H_{0}\left(2^{l} \omega\right)
$$

In other words, the filter is a lowpass filter with an approximate passband $|\omega| \in\left[0, \pi / 2^{J}\right]$. The filter relating the wavelet coefficients $\widetilde{b}_{j k}$ at level $j$ to the original signal has a frequency response

$$
B_{j}(\omega)=H_{1}\left(2^{J-j-1} \omega\right) \prod_{l=0}^{J-j-2} H_{0}\left(2^{l} \omega\right)
$$

which is a bandpass filter with an approximate passband $|\omega| \in\left[\pi / 2^{J-j}, \pi / 2^{J-j-1}\right]$ (Percival and Walden, 2000, pp. 96-97).

Let $F_{0}(\omega)$ be the frequency response of the lowpass synthesis filter. When $F_{0}(\omega)$ has $p$ zeros at $\pi$, the analysis wavelet has $p$ vanishing moments. Consequently, polynomials of degree $p-1$ can be expressed as a linear combination of the synthesis scaling functions $\phi_{0 k}(t)$. In order to filter out the linear trend in nonstationary economic time series such as real GDP, $p \geq 2$ is a necessary criteria in choosing wavelet filters.

\subsection{Biorthogonal $17 / 11$ wavelet filter}

In analyzing economic time series, the timing of events at various frequencies is important. This motivates the use of biorthogonal, rather than orthogonal, wavelet filters, which have linear phase (zero phase when centered). Within the family of biorthogonal filters, the filter must be sufficiently long to avoid undesirable artifacts in the filtered series. Based on experimentation, starting with shorter filters, I have found that the 17/11 filter works well in practice. The rest of this section highlights the relevant facts about this filter.

Figure 1 shows the impulse response of the analysis and synthesis filters in the biorthogonal 17/11 filter bank. The filters are symmetric, resulting in zero phase. The highpass analysis (synthesis) filter is the alternating flip of the lowpass synthesis (analysis) filter, assuring perfect reconstruction. The lowpass analysis filter $H_{0}(\omega)$ has 8 zeros at $\pi$, and the 
lowpass synthesis filter $F_{0}(\omega)$ has 6 zeros at $\pi$. The analysis wavelet therefore has 6 vanishing moments, and the synthesis scaling function can replicate polynomials of degree 5 .

Figure 2 shows the frequency response of the biorthogonal wavelet filter. Frequency is in units of cycles per period, which is radian frequency normalized by $2 \pi$. Then periodicity is simply the inverse of frequency. At level 0 approximation, the wavelet filter is a lowpass filter with a stopband of approximately 32 periods (see equation (9)). The level 0, 1, and 2 details correspond to bandpass filters of approximately $16-32,8-16$, and $4-8$ periods, respectively (see equation (10)). The level 3 detail corresponds to a highpass filter with a stopband of approximately 4 periods. Thus, a 4 -level wavelet decomposition is a natural way to filter an economic time series into trend (periodicity greater than 32 quarters), cycle (periodicity of 4-32 quarters), and noise (periodicity less than 4 quarters), whose sum is the original series by the perfect reconstruction property of the underlying filter bank (see equation 1$)^{2}$

\section{Measuring business cycles}

I now apply multiresolution wavelet analysis, using the biorthogonal 17/11 filter, to study the business-cycle component of real GDP. Figure 3 plots quarterly log real GDP and its long-run trend since 1947. The shaded regions of the figure correspond to recessions, from peak to trough, as determined by the National Bureau of Economic Research (NBER). The long-run trend is the level 0 approximation of the wavelet decomposition, corresponding to cycles with periodicity greater than 32 quarters. The plot shows that recessions are periods of sustained zero or negative growth in real GDP. Since the mid-1980's, GDP has not deviated far from its long-run trend.

The top three panels of Figure 4 shows the first three levels of detail in the wavelet decomposition. Levels 0 through 2 contain the business-cycle components of GDP, corresponding to 16-32, 8-16, and 4-8 quarter cycles. (Level 3 detail, which corresponds to high frequency

\footnotetext{
${ }^{2}$ Baxter and King (1999) define the business cycle as cycles with periodicity of 6-32 quarters. Since the scales in wavelet filters are dyadic, I use 4 instead of 6 quarters in the definition. The difference is obscured in practice because these finite impulse response filters are only approximations to ideal brick wall filters.
} 
noise with cycles less than 4 quarters, is not reported.) The wavelet analysis reveals interesting changes in the volatility of the business-cycle component at various scales. At the scale corresponding to 16-32 quarter cycles, the largest business-cycle fluctuations occurred in the 1970's through the early 1980's, with deviations exceeding $2.5 \%$ of the long-run trend. At higher scales corresponding to 8-16 and 4-8 quarter cycles, the largest volatility occurred before the 1960's. Since the mid-1980's, the business-cycle fluctuations of GDP have been relatively small. The source of the recent decline in business-cycle volatility is a subject of current debate (see Stock and Watson, 2003, and references therein).

The sum of the three business-cycle components is shown in the bottom panel of Figure 4. As a comparison, the figure also shows real GDP filtered by the approximate bandpass filter. The two filtered series are remarkably similar. The local minima of the filtered series generally correspond to the NBER troughs. The peaks of the filtered series usually occur a few quarters before the NBER peak, which is a consequence of the slowdown in GDP growth before the onset of a recession. The only recession that both the wavelet filter and the bandpass filter miss is the 1980 recession, which began in January and ended in July. First, the duration of this recession is short. Second, Figure 3 shows that GDP was well above the long-run trend before the slowdown, so GDP did not fall much below the trend during the recession.

\section{References}

Baxter, M., King, R. G., 1999. Measuring business cycles: Approximate band-pass filters for economic time series. Review of Economics and Statistics 81 (4), 575-593.

Kim, C.-J., Nelson, C. R., 1999. Has the U.S. economy become more stable? A Bayesian approach based on a Markov-switching model of the business cycle. Review of Economics and Statistics 81 (4), 608-616.

McConnell, M. M., Perez-Quiros, G., 2000. Output fluctuations in the United States: What has changed since the early 1980's? American Economic Review 90 (5), 1461-1476. 
Percival, D. B., Walden, A. T., 2000. Wavelet Methods for Time Series Analysis. Cambridge Series in Statistical and Probabilistic Mathematics. Cambridge University Press, Cambridge.

Stock, J. H., Watson, M. W., 2003. Has the business cycle changed and why? In: Gertler, M., Rogoff, K. (Eds.), NBER Macroeconomics Annual 2002. MIT Press, Cambridge, MA.

Strang, G., Nguyen, T., 1997. Wavelets and Filter Banks. Wellesley-Cambridge Press, Wellesly, MA. 

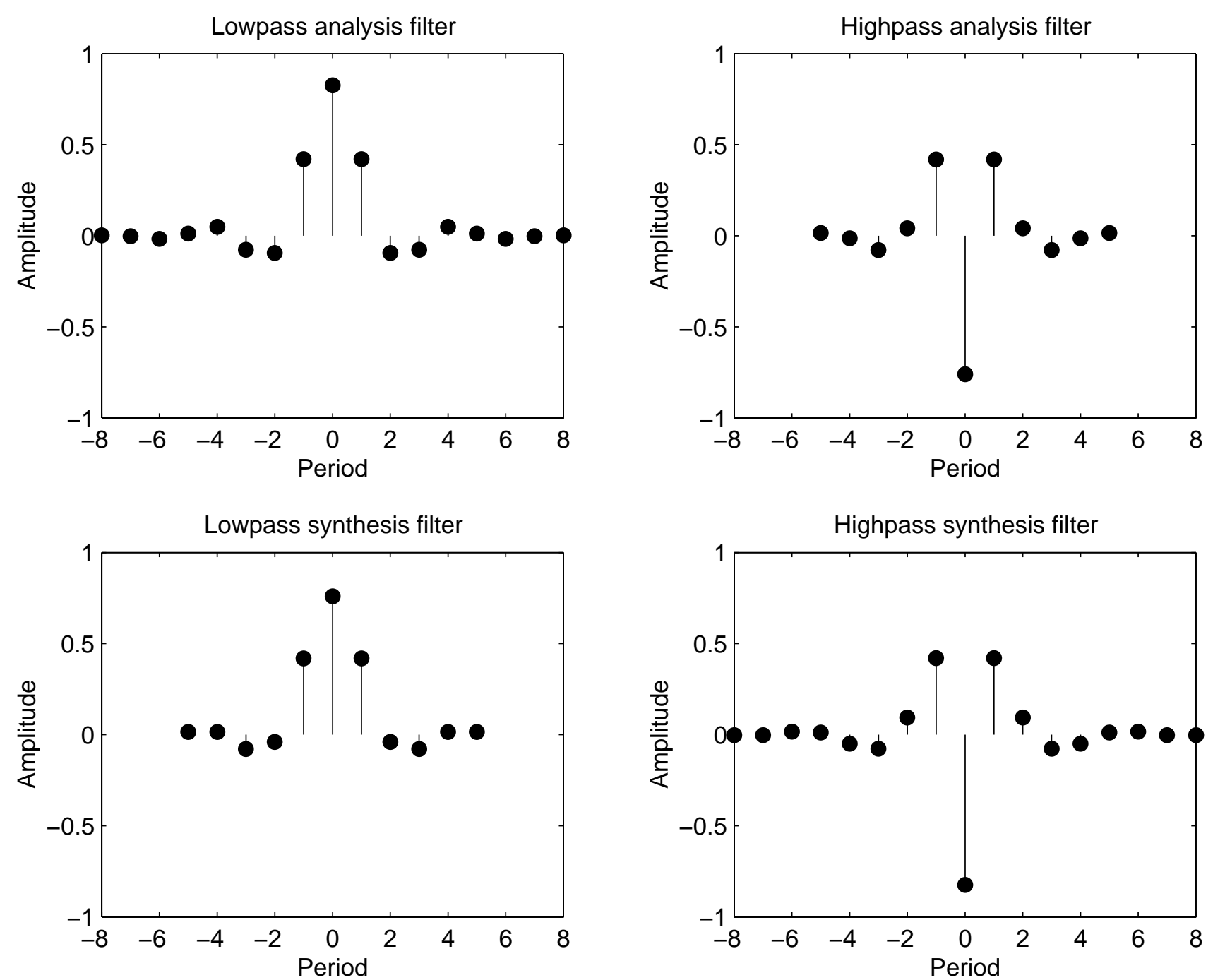

Figure 1: Impulse response of the analysis and synthesis filters in the biorthogonal 17/11 filter bank. 

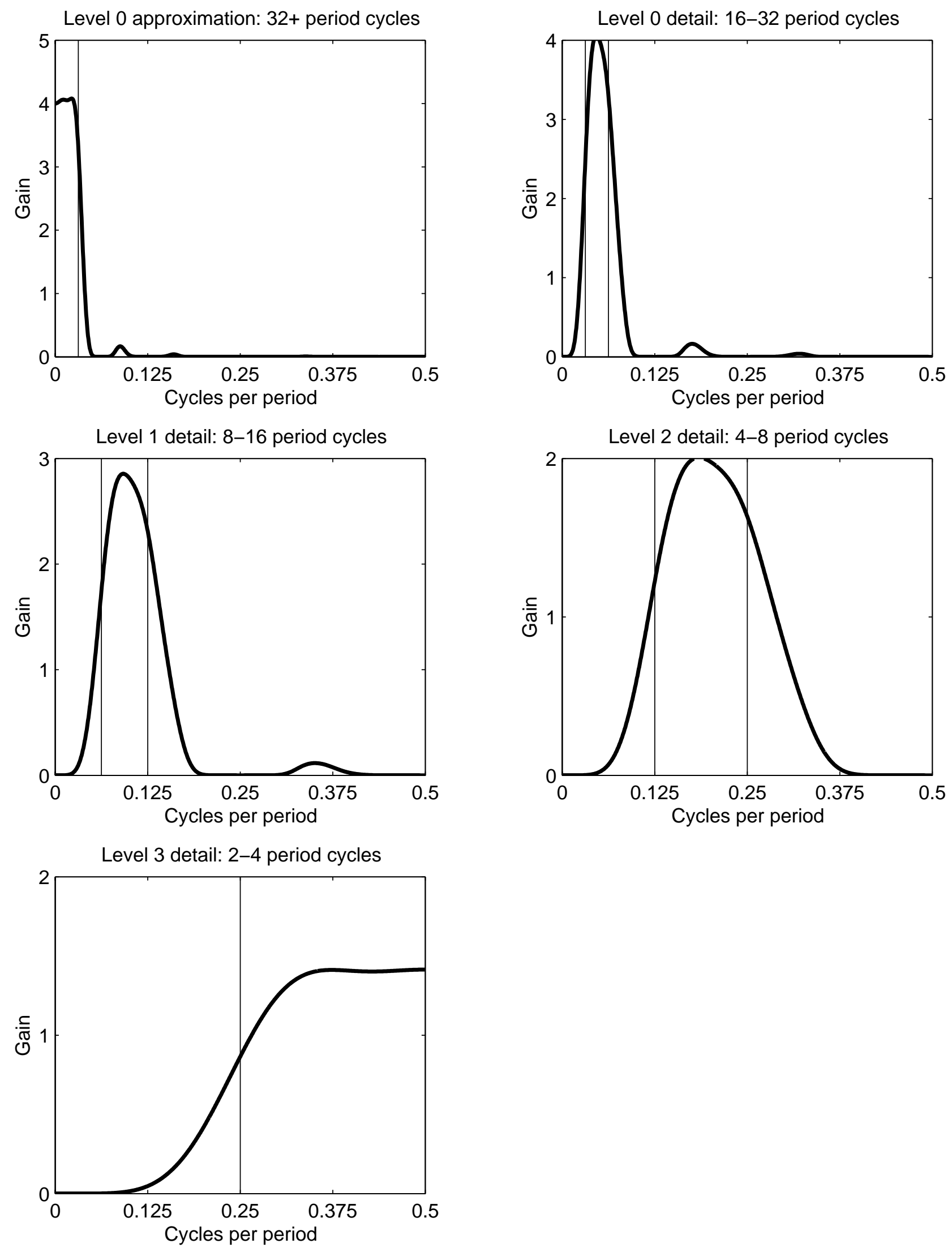

Figure 2: Frequency response of the biorthogonal 17/11 wavelet filter. 


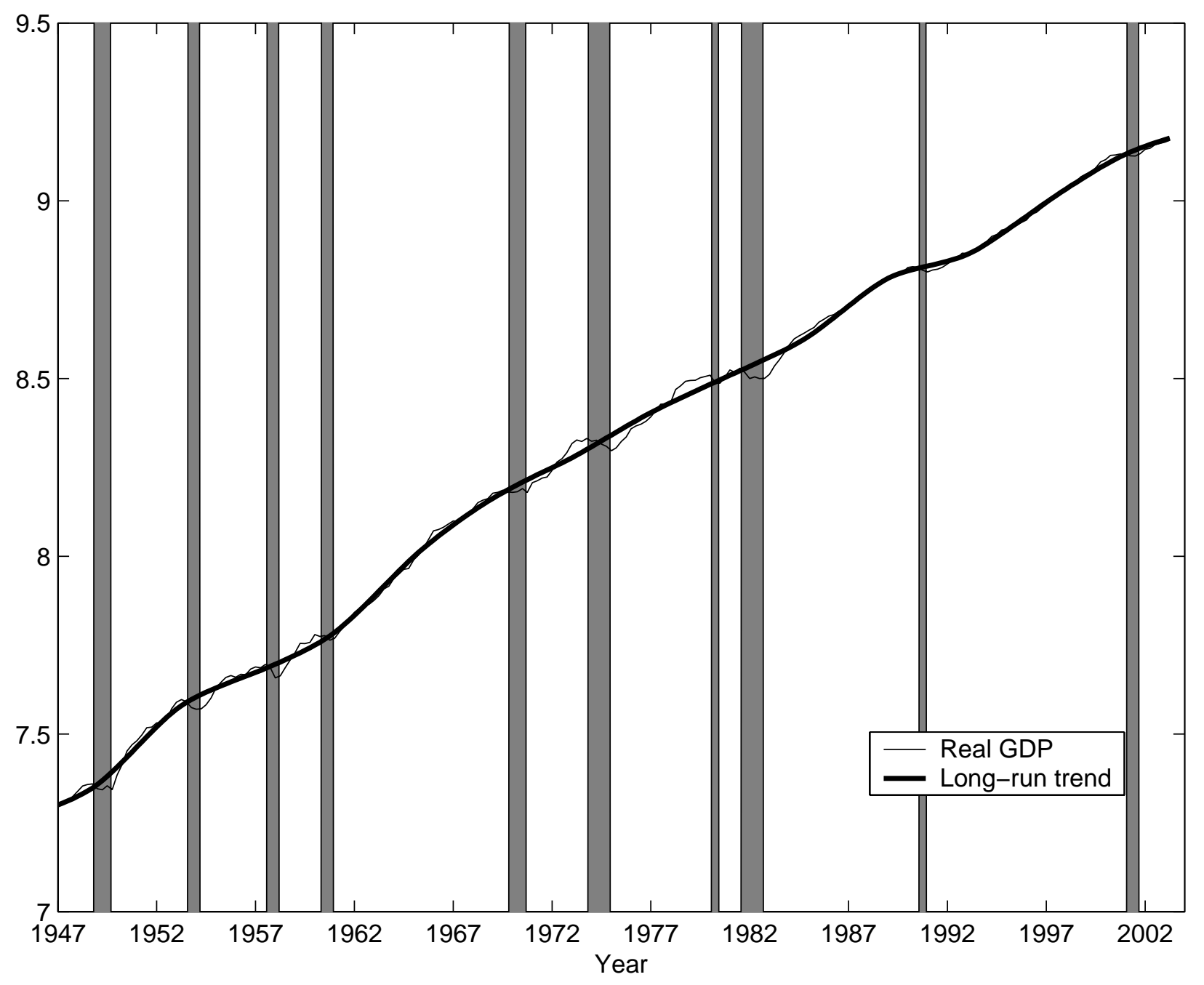

Figure 3: Log real GDP and long-run trend. Shaded regions are NBER recessions. 
Level 0 detail: $16-32$ quarter cycles

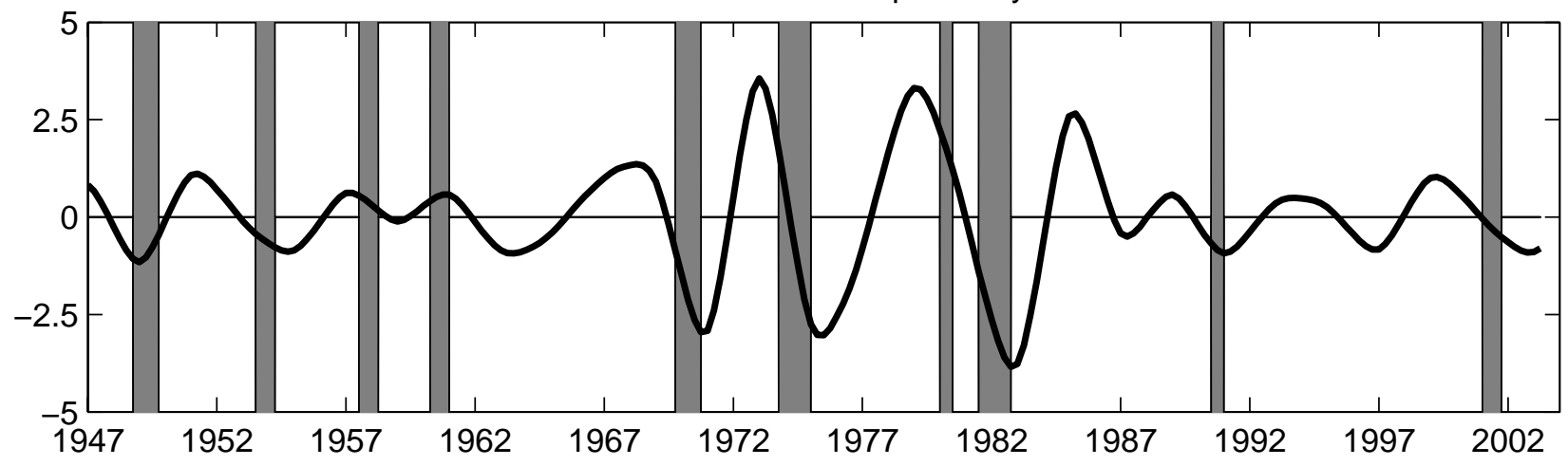

Level 1 detail: 8-16 quarter cycles

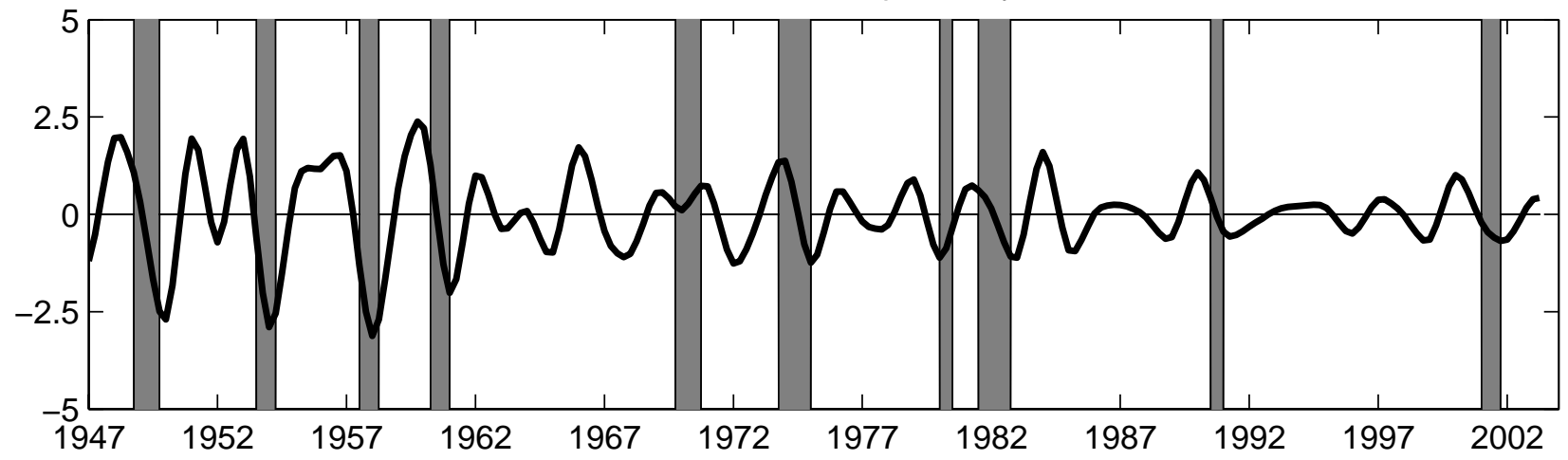

Level 2 detail: 4-8 quarter cycles

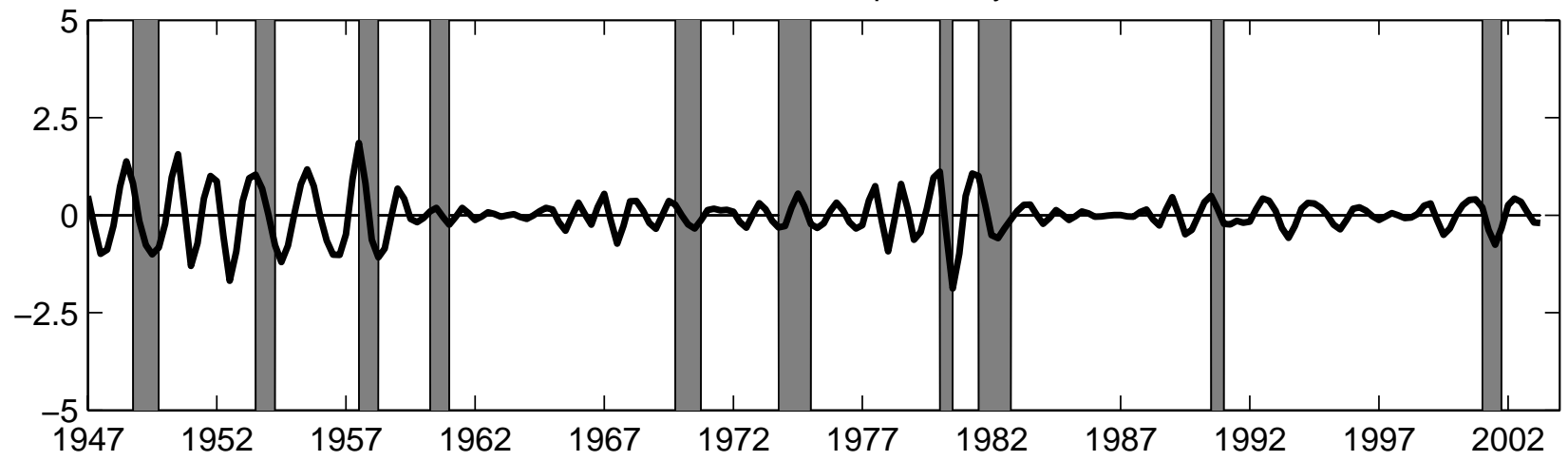

Levels $0-3$ detail: $4-32$ quarter cycles

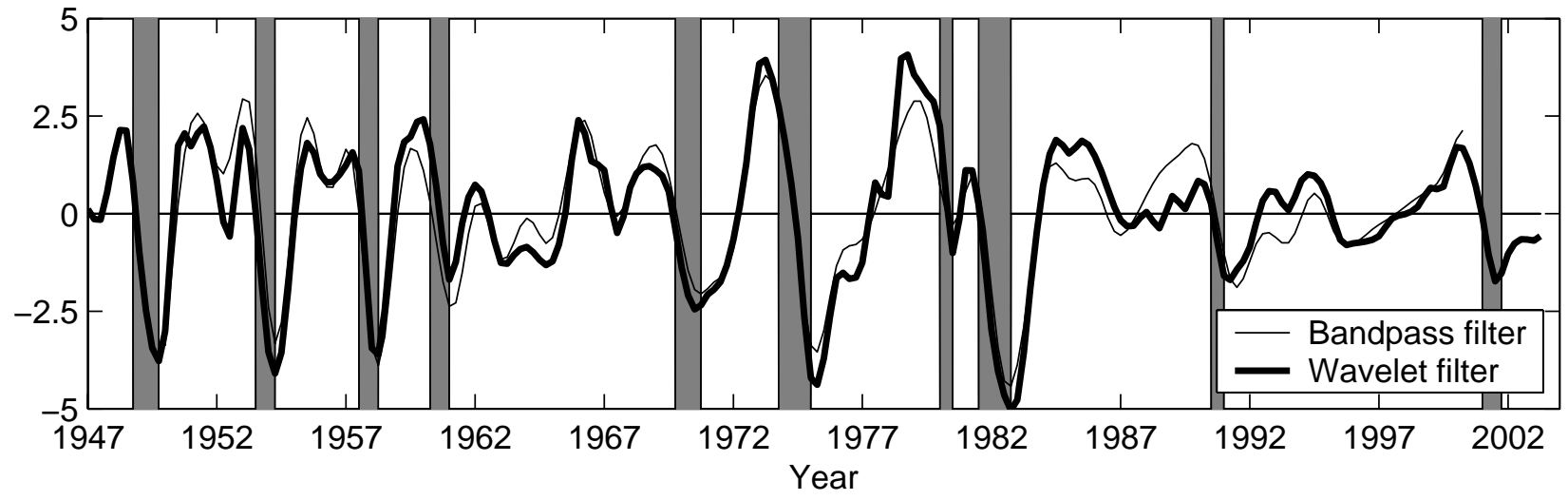

Figure 4: Business-cycle component of log real GDP, measured as percent deviation from the long-run trend. Shaded regions are NBER recessions. 